\title{
Regulation of IL-10 by Chondroitinase ABC Promotes a Distinct Immune Response following Spinal Cord Injury
}

\author{
Athanasios Didangelos, Michaela Iberl, Elin Vinsland, Katalin Bartus, and Elizabeth J. Bradbury \\ Wolfson Centre for Age Related Diseases, Guy's Campus, King's College London, London SE1 1UL, United Kingdom
}

Chondroitinase $\mathrm{ABC}(\mathrm{Ch} \mathrm{ABC}$ ) has striking effects on promoting neuronal plasticity after spinal cord injury (SCI), but little is known about its involvement in other pathological mechanisms. Recent work showed that ChABC might also modulate the immune response by promoting M2 macrophage polarization. Here we investigate in detail the immunoregulatory effects of ChABC after SCI in rats. Initially, we examined the expression profile of $16 \mathrm{M} 1 / \mathrm{M} 2$ macrophage polarization markers at $3 \mathrm{~h}$ and $7 \mathrm{~d}$ postinjury. ChABC treatment had a clear effect on the immune signature after SCI. More specifically, ChABC increased the expression of the anti-inflammatory cytokine IL-10, accompanied by a reduction in the proinflammatory cytokine IL-12B in injured spinal tissue. These effects were associated with a distinct, IL-10-mediated anti-inflammatory response in ChABC-treated spinal cords. Mechanistically, we show that IL-10 expression is driven by tissue injury and macrophage infiltration, while the p38 MAPK is the central regulator of IL-10 expression in vivo. These findings provide novel insights into the effects of $\mathrm{ChABC}$ in the injured spinal cord and explain its immunoregulatory activity.

Key words: chrondroitinase ABC; inflammation; macrophage polarization; proteoglycans; spinal cord injury

\section{Introduction}

After spinal cord injury (SCI), the initial tissue damage is followed by dysregulated inflammation and reactive gliosis (Silver and Miller, 2004). The accumulation of chondroitin sulfate proteoglycans (CSPGs) in the glial scar inhibits regeneration and limits functional repair (Fawcett and Asher, 1999). Previous work from our laboratory and others has shown that the glycolytic enzyme chondroitinase $\mathrm{ABC}$ (ChABC) removes glycosaminoglycan (GAG) chains from glial scar CSPGs, and promotes neuronal regeneration and plasticity after SCI in vivo (Bradbury et al., 2002; Alilain et al., 2011; Burnside and Bradbury, 2014). Recently, we found that the beneficial effects of ChABC are not limited to enhanced plasticity. Large-scale CSPG deglycosylation via viral overexpression of ChABC was associated with an increase in anti-inflammatory M2 macrophages and reduced tissue pathology (Bartus et al., 2014). In the spinal cord, alternative (M2) macrophage activation could

Received July 17, 2014; revised 0ct. 6, 2014; accepted 0ct. 28, 2014.

Author contributions: A.D. and E.J.B. designed research; A.D., M.I., and E.V. performed research; A.D., E.V., and K.B. analyzed data; A.D. and E.J.B. wrote the paper.

This project was funded by the Medical Research Council (Senior Non-Clinical Fellowship Award G1002055 to E.J.B.) and the Rosetrees Trust (Grant JS16/M276 to E.J.B. and A.D.). We would like to thank Professor Joost Verhaagen for provision of lentiviral vector chondroitinase.

The authors declare no competing financial interests.

This article is freely available online through the J Neurosci Author Open Choice option.

Correspondence should be addressed to either Elizabeth J. Bradbury or Athanasios Didangelos, Wolfson

Centre for Age Related Diseases, Guy's Campus, King's College London, London SE1 1UL, UK. E-mail: elizabeth.bradbury@kcl.ac.uk or athanasios.didangelos@kcl.ac.uk.

DOI:10.1523/JNEUROSCI.2927-14.2014

Copyright $\odot 2014$ Didangelos et al.

This is an Open Access article distributed under the terms of the Creative Commons Attribution License (http://creativecommons.org/licenses/by/3.0), which permits unrestricted use, distribution and reproduction in any medium provided that the original work is properly attributed. limit secondary inflammatory injury and promote tissue repair (Kigerl et al., 2009; David and Kroner, 2011). Here, we set out to investigate the immunomodulatory effects of $\mathrm{ChABC}$ in detail.

\section{Materials and Methods}

Animals. Adult female Sprague Dawley rats ( 200 g; Harlan Laboratories) were used for all experiments and were housed under a $12 \mathrm{~h} \mathrm{light/}$ dark cycle with ad libitum access to food and water. All procedures were performed in accordance with the United Kingdom Animals (Surgical Procedures) Act 1996.

Spinal cord injury. Anesthetized (ketamine $60 \mathrm{mg} / \mathrm{kg}$, and medetomidine $0.25 \mathrm{mg} / \mathrm{kg}$ ) rats received a midline $150 \mathrm{kdyne}$ spinal contusion injury at spinal level T10/T11 using an Infinite Horizon Impactor (Precision Systems Instrumentation), as previously described (James et al., 2011). Animals were kept for either 3 h or $7 \mathrm{~d}$ postinjury. Spinal cord tissue was then used for biochemical [mRNA, protein, and conditioned medium (CM)] analyses.

Intrathecal injections by lumbar puncture. To achieve sufficient digestion of spinal cord CSPGs, we delivered high-dose ChABC in large volumes $(100 \mu \mathrm{l})$ via multiple lumbar puncture injections. Although less potent than viral ChABC overexpression (Bartus et al., 2014), this regime leads to more effective CSPG digestion than previous intrathecal paradigms (Barritt et al., 2006). Animals received intrathecal injections by lumbar puncture at the intervertebral space, of either PBS (supplemented with glucose) or high-dose protease-free ChABC dissolved in PBS with glucose (Sigma; $0.5 \mathrm{U}$ in $100 \mu$ linjection volume). We used the following three injection regimes. (1) Animals were injected twice at 48 and $24 \mathrm{~h}$ before SCI. Injured animals were then kept for $3 \mathrm{~h}$ before culling. (2) Animals were injected every $48 \mathrm{~h}$ over $7 \mathrm{~d}$ after SCI (with the first injection at $1 \mathrm{~h}$ postinjury). Injured animals were then kept for $7 \mathrm{~d}$ before culling. For IL-10 neutralization, ChABC was mixed with rabbit anti-IL-10 neutralizing antibodies ( $5 \mu \mathrm{g}$; Abcam) or $5 \mu \mathrm{g}$ of rabbit isotype antibodies (Abcam). (3) The effect of intrathecal administration of 
high-dose $\mathrm{ChABC}$ was also tested on intact cords. Animals were injected twice at 48 and $24 \mathrm{~h}$ before culling.

Peritoneal macrophages. Macrophages were extracted from the peritoneum of adult female rats $(250-350 \mathrm{~g})$. Animals were killed in a $\mathrm{CO}_{2}$ chamber. The peritoneal cavity was infused with $60 \mathrm{ml}$ of PBS. Five minutes later, peritoneal fluid was aspirated via a midline incision $2 \mathrm{~cm}$ caudal to the xiphoid process. Cells were spun for $7 \mathrm{~min}$ at $1100 \mathrm{rpm}$. Pellets were dissolved in culture medium (DMEM, antibiotics, and 15\% FCS) and plated to confluency ( $\sim 250,000$ cells/well in 96-well plates). Cells were cultured at $37^{\circ} \mathrm{C}$ and $5 \% \mathrm{CO}_{2}$. After $72 \mathrm{~h}$, cells were serum starved in plain DMEM for $4 \mathrm{~h}$ before stimulation. Each experimental condition or time point was performed in duplicate for Western blotting or in quadruplicate for mRNA analysis.

Conditioned medium. Conditioned medium from injured PBS- or ChABC-injected spinal cords was prepared in serum-free DMEM, as described by Bartus et al. (2014).

p38 inhibition in vivo. p38 MAPK activity was blocked using the highly specific MAPK-activated protein kinase-2 blocker SB203580. The inhibitor specifically targets $\mathrm{p} 38$ (p38 $\alpha$ and $\mathrm{p} 38 \beta$ ) even at concentrations $>10$ $\mu \mathrm{M}$ (Saklatvala et al., 1996). For spinal inhibition of $\mathrm{p} 38$, anesthetized animals received two intrathecal injections of SB203580 (10 $\mu \mathrm{M}$ in PBS/ $0.01 \%$ DMSO), 60 and 30 min before a 150 kdyne contusion SCI. Control cords were injected with PBS/0.01\% DMSO only. For systemic inhibition of $\mathrm{p} 38$, animals received three intraperitoneal injections of SB203580 (5 $\mu \mathrm{m} / \mathrm{kg}$ in PBS/0.01\% DMSO; $0.5 \mathrm{ml}$ injection volume), at 24,12 , and $0.5 \mathrm{~h}$ before SCI, and once immediately after injury.

Protein extraction and immunoblotting. Protein extraction and immunoblotting from injured or intact spinal cord segments was performed as described by Bartus et al. (2014). The following antibodies were used: rabbit cyclooxygenase 2 (COX2), IRF5, and IL-10, mouse CD68, brevican, and $\beta$-actin loading control (Abcam); goat arginase-1 (ARG1; Santa Cruz Biotechnology); CD206 (R\&D Systems); rabbit IBA1 (ionized calcium-binding adaptor molecule 1; Wako); aggrecan (Millipore Bioscience Research Reagents); mouse chondroitin-4-sulfate (C4S; AbD Serotec); rabbit GAPDH and mouse neurocan (Sigma); and rabbit pp38, pERK, pSTAT3, pATF2, and mouse I $\kappa \mathrm{B} \alpha$ (Cell Signaling Technology).

RNA extraction and quantitative PCR. Real-time PCR was performed using prevalidated TaqMan rat primer/probes (Invitrogen) in an automated Roche thermocycler. The fold change in mRNA expression of target genes in injured and treated samples $(\sim 6 \mathrm{~mm}$ from T10 spinal cord) was calculated relative to appropriate controls using the $\Delta \Delta \mathrm{Ct}$ method. GAPDH served as the housekeeping gene. All short amplicon primer/probes were designed, tested, and recommended by Applied Biosystems as they detect the maximum number of transcripts for the gene of interest, they do not detect homologs, they sequence across an exonexon junction, they do not hybridize with multiple off-target genes, and they do not target the 5' UTR.

Immunohistochemistry. T10/T11spinal cord segments $(\sim 1 \mathrm{~cm})$ were collected from perfused-fixed animals that received intrathecal injections of either PBS or ChABC. Cryosections were probed with mouse CD68 (1:100 dilution; Abcam) and C4S (1:100 dilution; Millipore Bioscience Research Reagents) antibodies. Sections were incubated for $16 \mathrm{~h}$ at $4^{\circ} \mathrm{C}$ before applying goat anti-mouse Alexa Fluor 568 (red) for $90 \mathrm{~min}$. Sections were washed and mounted using VectaStain medium supplemented with DAPI for nuclear staining.

\section{Results}

\section{IL-10 mRNA is upregulated early after SCI}

To examine inflammatory activation in the injured spinal cord, adult rats received a clinically relevant contusion injury at spinal level T10, using an automated impact device (Infinite Horizon Impactor; James et al., 2011). To determine the effect of proteoglycan deglycosylation on the acute inflammatory response, one group of animals received two intrathecal injections of highconcentration ChABC ( 0.5 U/injection) at 48 and $24 \mathrm{~h}$ before injury. Control animals were injected with PBS. Inflammatory activation was monitored in the injury epicenter using quantita- tive mRNA expression (TaqMan Assay) at $3 \mathrm{~h}$ postinjury (Fig. $1 A)$. We focused on genes associated with M1 and M2 macrophage subtypes (for review, see Gordon and Martinez, 2010; Mantovani et al., 2013). Most genes were rapidly upregulated after SCI in both PBS- and ChABC-injected cords (Fig. 1A). Pretreatment with $\mathrm{ChABC}$ caused a clear upregulation of IL-10 mRNA and a concomitant reduction in the expression of IL-12B (Fig. $1 A, C$ ). Apart from the IL-10 high/IL-12B low effect, which is characteristic of M2 macrophage polarization (Mantovani et al., 2013), ChABC treatment altered the expression of several genes compared with PBS injections, but did not produce a clear distinction between M1 and M2 markers. For instance, while it increased the expression of classic M2 markers CD206, CD163, and ARG1, it also enhanced the expression of CD32 and inducible nitric oxide synthase (iNOS; $p<0.05$; Fig. $1 A$ ), typically associated with M1 macrophages (Kigerl et al., 2009). ChABCtreated cords also showed increased expression of IBA1, expressed by macrophages and microglia, and the leukocyte common antigen CD45 (Fig. 1A,C). The upregulation of CD45 and IBAl at this early time point $(3 \mathrm{~h})$ suggested increased infiltration of immune cells in ChABC-treated cords acutely after injury. The sustained differential regulation of IL-10 and IL-12B was observed in another cohort of animals that first received spinal T10 contusion and subsequent injections of either PBS or ChABC for $7 \mathrm{~d}$ (Fig. 1 B, D). M2-associated genes CD206, CD163, ARG1, and heme oxygenase 1 (HMOX1) were upregulated in ChABC-treated cords, together with M1-associated genes CXCL10 and iNOS $(p<0.05$; Fig. $1 B)$. CD45 and IBA1 were, instead, unchanged (Fig. $1 B, D)$.

\section{Inflammatory protein changes and proteoglycan remodeling}

We then examined the effects of injury and ChABC treatment on inflammatory protein markers and extracellular matrix proteoglycans in another group of animals. Seven days postinjury, COX2 and CD68 were upregulated in the injury epicenter of both PBS- and ChABC-injected cords, suggesting that the general inflammatory status was similar with both treatments (Fig. $1 E$ ). In contrast, ARG1 and IBA1 showed a small but significant increase in expression with ChABC treatment compared with PBS treatment (Fig. $1 E, F$ ). The upregulation of arginase 1 is noteworthy since it is an M2 marker and a classic IL-10 response gene (Mantovani et al., 2013). Elevated levels of IBA1 protein $7 \mathrm{~d}$ postinjury in ChABC-treated animals is likely due to increased early IBA1 mRNA (Fig. 1C). mRNA transcripts have a shorter half-life than proteins, which tend to turn over slower and accumulate while mRNA has returned to baseline levels. IRF5, a transcription factor involved in M1 macrophage polarization (Krausgruber et al., 2011), was downregulated in the injury epicenter with both treatments (Fig. 1E). Moreover, ChABC treatment caused a clear loss in the immunoreactivity of glycosylated neurocan $(\sim 260 \mathrm{kDa})$ in both the injury epicenter and in intact tissue rostral to the epicenter (Fig. 1E,F). This decrease does not necessarily reflect a reduction in neurocan core protein expression; indeed, in a previous study (Bartus et al., 2014) using an antibody that recognizes neurocan core protein $(\sim 150 \mathrm{kDa})$ we did not see a reduction at the protein level after viral ChABC delivery. Aggrecan was increased in intact rostral tissue of ChABC-treated animals (Fig. $1 E, F$ ), while brevican was unaffected (Fig. $1 E$ ). ChABC activity in cords was confirmed by immunoblotting for deglycosylated C4S GAG stubs (Fig. 1E). 

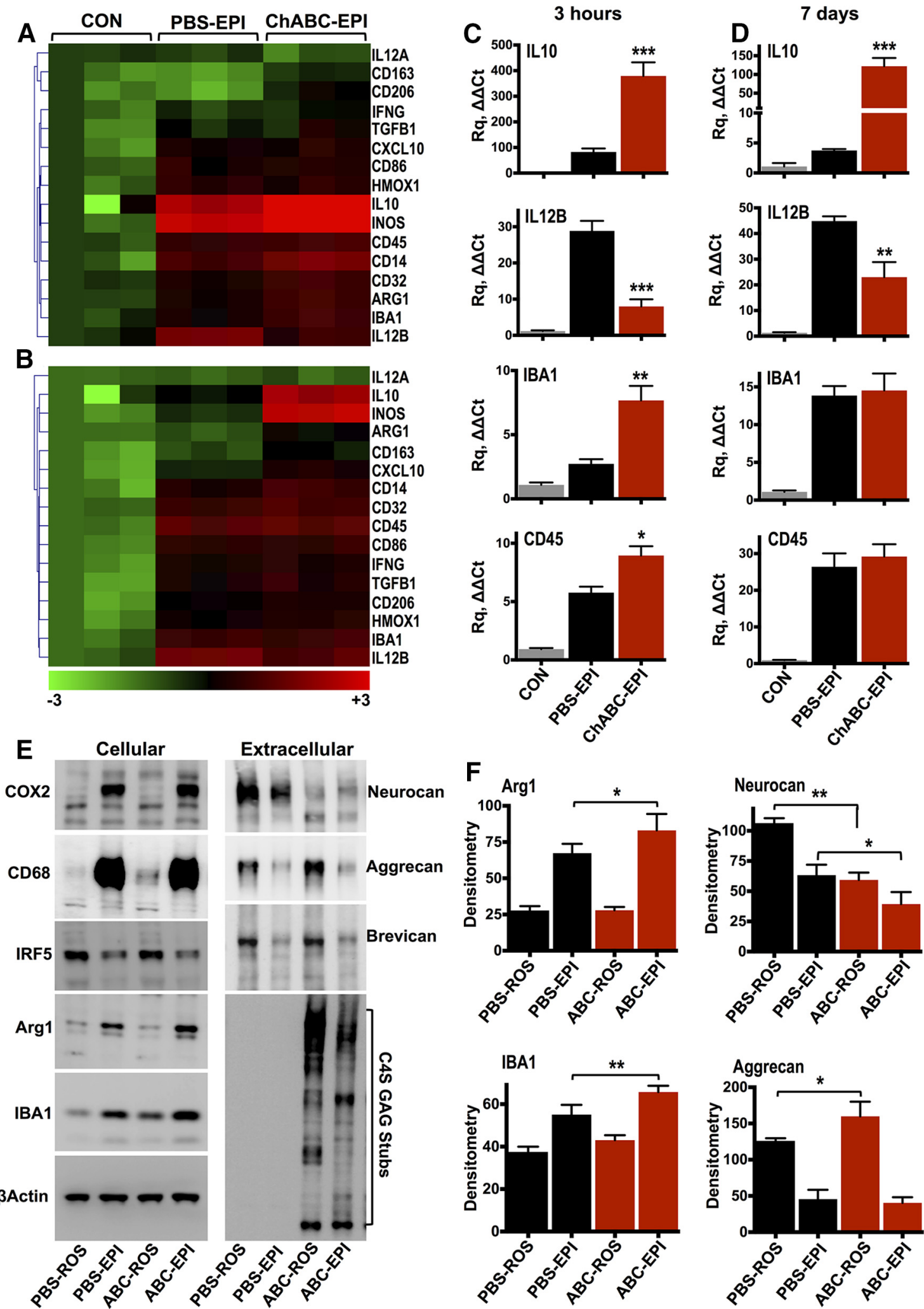

Figure 1. ChABC treatment alters the inflammatory response after $S C I . A, B$, Heat maps display normalized $[-3$ (green) to +3 (red)] differences in relative gene expression quantified by TaqMan quantitative PCR in the injury epicenter (EPI) of either PBS- or ChABC-injected cords $3 \mathrm{~h}(\boldsymbol{A})$ and $7 \mathrm{~d}(\boldsymbol{B})$ postinjury. Fold change in mRNA expression was calculated using the $\Delta \Delta C \mathrm{Ct}$ method, relative to control (CON) T10 spinal cord collected from uninjured animals. GAPDH served as the housekeeping gene. Statistical analysis was performed using one-way ANOVA and, for multiple comparisons, using he Holm-Sidak test (PBS-EPI vs ChABC-EPI). C, D, Graphs depict quantitative PCR relative quantitation (Rq $\Delta \Delta C \mathrm{Ct}$ ) for IL-10, IL-12B, IBA1, and CD45 in control, PBS-injectd, and ChABC-injected cords $3 \mathrm{~h}(\boldsymbol{C})$ and $7 \mathrm{~d}(\boldsymbol{D})$ postinjury. Results are reported as the mean fold change in gene expression \pm SEM, $n=3$ animals per group. Statistical analysis was performed using ANOVA, and for multiple comparisons the Holm-Sidak test (PBS-EPI vs ChABC-EPI). ${ }^{*} p<0.05,{ }^{* *} p<0.01,{ }^{* * *} p<0.001$. E, Cellular and extracellular protein levels were measured by Western blotting of the T10 injury EPI of PBS- and ChABC-injected spinal cords, $7 \mathrm{~d}$ postinjury. $F$, Intact tissue was collected from rostral (ROS) $T 1$ spinal cord of the same animals to control for protein changes caused by injections on uninjured tissue. Protein levels were quantified by densitometry $(\boldsymbol{F})$. Results are mean density values $\pm \mathrm{SEM}, n=3$ animals per group. Statistical analysis was performed using ANOVA, and for multiple comparisons using the Holm-Sidak test. ${ }^{*} p<0.05,{ }^{* *} p<0.01$. 


\section{IL-10 neutralization affects macrophage inflammatory markers}

In IL-10 blocking experiments, we examined the effect of neutralization of spinal cord-derived IL-10 on the expression of macrophage phenotypic and inflammatory markers (Fig. 2). Injury epicenter (T10) explants were dissected ex vivo from contused rats injected with $\mathrm{PBS}$ or $\mathrm{ChABC}$ for $7 \mathrm{~d}$ postinjury. Injury epicenter explants were then cultured in serum-free medium for $16 \mathrm{~h}$ to allow release of soluble inflammatory factors. The CM was then used to stimulate resting rat peritoneal macrophages in the presence of either IL-10 neutralizing antibodies $(+\mathrm{Nt})$ or isotype control IgG $(-\mathrm{Nt})$. Neutralization of IL-10 resulted in a small but significant reduction of CD206 on macrophages treated with conditioned medium from $\mathrm{ChABC}$-injected injured spinal cords (Fig. $2 A, B$ ). Arginase 1 was also decreased after IL-10 neutralization in both PBS and ChABC conditioned medium, while baseline phosphorylation of the transcription factor STAT3 was abolished (Fig. 2A,B). Activation of STAT3 by IL-10 is central for cytokine suppression in macrophages (Williams et al., 2007). COX2 was strongly upregulated by both PBS and ChABC conditioned medium, but was unaffected by IL-10 neutralization (Fig. 2A). Macrophages treated with $\mathrm{ChABC}$ conditioned medium showed a reduction in IRF5 after IL-10 neutralization (Fig. 2A), although the reduction was not statistically significant.

We then examined mRNA expression of inflammatory genes typically inhibited by IL-10. IL-12B and iNOS were significantly upregulated after IL-10 neutralization, together with the inflammatory cytokines IL- $1 \beta$, TNF- $\alpha$, and IL-6 (Fig. $2 C$ ). In contrast, the M2 phenotypic marker CD163 was decreased (Fig. 2C). Although neutralization of IL-10 in the conditioned medium had a clear effect on macrophage gene expression, it did not differentiate between PBS- and ChABC-injected cords, with the exception of CD206 (Fig. 2A,B). Most likely, some IL-10 is present in conditioned medium from PBS-treated cords, while cultured macrophages evidently express IL-10 at baseline. The expression of CD206 was next investigated in vivo in contused rats treated with $\mathrm{ChABC}$ and intrathecal administration of IL-10neutralizing antibodies for $7 \mathrm{~d}$ (Fig. 2D). Compared with animals treated with isotype control IgG, IL-10 neutralization resulted in a significant reduction in CD206 mRNA (Fig. 2D), while iNOS showed a decreasing trend $(p=0.06)$. All other inflammatory markers examined (IL- $1 \beta$, TNF- $\alpha$, IL-6, and IL-12B) were unaffected by in vivo IL-10 neutralization (Fig. $2 D$ ). At the protein level (Fig. $2 E, F$ ), we found a significant reduction in arginase 1 , as well as in GFAP, neuronal $\beta 3$ tubulin, and myelin basic protein (MBP), indicating increased cellular toxicity after IL-10 neutralization.

\section{IL-10 regulation by in vitro injury and $\mathrm{Ch} A \mathrm{AC}$ injection}

The regulation of IL-10 in the spinal cord was next investigated in more detail in a tissue explant in vitro injury preparation. To determine whether IL-10 is expressed as a result of the mechanical injury to the spinal cord, intact cords were injured in vitro. To ensure that blood cells did not affect gene expression, animals were perfused with PBS before spinal cord dissection. T10 explants were diced on a Petri dish and cultured for $3 \mathrm{~h}$ (in vitro injury). Control explants were collected from the adjacent T9 spinal cord segment and were snap frozen immediately upon dissection to determine baseline mRNA levels (Fig. 3A). Three genes were significantly increased after in vitro injury: iNOS, IL10 , and IL-12B ( $p<0.05$; Fig. $3 A$ ). Thus, IL-10 can be rapidly induced in spinal cords by simple mechanical injury, even in the absence of vascular supply. Gene expression could be due either to the release of soluble factors or direct mechanosensing of resident cells, most likely microglia and astrocytes (Ledeboer et al., 2002).

We then tested whether intrathecal ChABC injections had a direct effect on intact spinal cords. ChABC injections in uninjured animals caused a significant induction in IL-10 mRNA, together with an increase in levels of IBA1, CD14, CD45, and the macrophage chemokine receptor CCR2 (Fig. $3 B$ ). The level of IL-12B was unchanged (Fig. $3 B$ ). These data suggest macrophage infiltration, although de novo expression of these markers by resident cells cannot be excluded. Immunofluorescence confirmed proteoglycan deglycosylation after ChABC injections (C4S, GAG stubs; Fig. $3 C$ ) and revealed the accumulation of CD68-positive cells in the anterior spinal and sulcal vessels (Fig. $3 D$ ), and in the vasocorona and its branches (Fig. $3 E$ ), providing further evidence of macrophage infiltration with $\mathrm{ChABC}$ treatment. When we combined intrathecal ChABC injections with in vitro injury, IL-10 expression was strongly upregulated in explants dissected from ChABC-injected cords (Fig. 3F, In vitro Injury). In comparison, IL-12B was decreased, mirroring the IL-10 high/IL-12B low effect seen previously in contused tissue (Fig. $1 A-D$ ). Furthermore, when intact spinal cords were injected with ChABC that was previously boiled for $15 \mathrm{~min}$ to destroy enzymatic activity, the increase in IL-10 expression was diminished (Fig. 3G). We next treated contused rats with intraspinal injections of high-titer lentiviral vectors expressing ChABC (LV-ChABC; Fig. $3 H$; Bartus et al., 2014). Compared with control GFPexpressing vectors, LV-ChABC-injected cords showed a significant increase in IL-10 mRNA 7 d postinjury. Collectively, these results suggest that the upregulation of IL-10 is due to ChABC activity in the cord.

\section{IL-10 is regulated by $\mathrm{p} 38$ in the spinal cord}

The ERK and p38 MAPK and nuclear factor- $\kappa \mathrm{B}(\mathrm{NF}-\kappa \mathrm{B})$ signaling pathways are involved in the regulation of IL-10 expression in different systems (Saraiva and O'Garra, 2010). In experiments examining upstream signaling activation, conditioned medium sampled from injured T10 spinal cord explants caused rapid activation of the $\mathrm{p} 38$ and ERK MAPKs on resting peritoneal macrophages (Fig. $4 A, B$ ). The conditioned medium also activated the AP1 transcription factor ATF2 and caused the degradation of $\mathrm{I} \kappa \mathrm{B} \alpha$ (Fig. $4 A, B$ ), indicating activation of NF- $\kappa \mathrm{B}$. Conditioned medium from $\mathrm{ChABC}$-injected injured cords further enhanced the phosphorylation of p38 and, to a lesser extent, ATF2 (Fig. $4 A, B)$. In in vivo experiments, intrathecal delivery of the p38 inhibitor SB203580 (Saklatvala et al., 1996) before T10 contusion, did not affect the expression of either IL-10 or IL-12B $3 \mathrm{~h}$ postinjury (Fig. 4C). In contrast, systemic injections (intraperitoneal) of the inhibitor before and immediately after injury, led to a significant reduction in IL-10 expression (Fig. 4D). IL-12B, IBA1, and CD45 were not significantly affected (Fig. 4D). These results suggest, first, that IL-10 mRNA is regulated by p38 in vivo after SCI and, second, that immune cells infiltrating the injury site are responsible for most of the IL-10 expression.

\section{Discussion}

In this study, we provide novel insights into the immunomodulatory effects of ChABC after SCI. We describe the upregulation of IL-10, an anti-inflammatory factor that mediates cytokine suppression by transcriptional and post-transcriptional regulation of its target genes (Williams et al., 2007). Treatment with ChABC caused a mixed regulation of M1/M2 macrophage markers. 

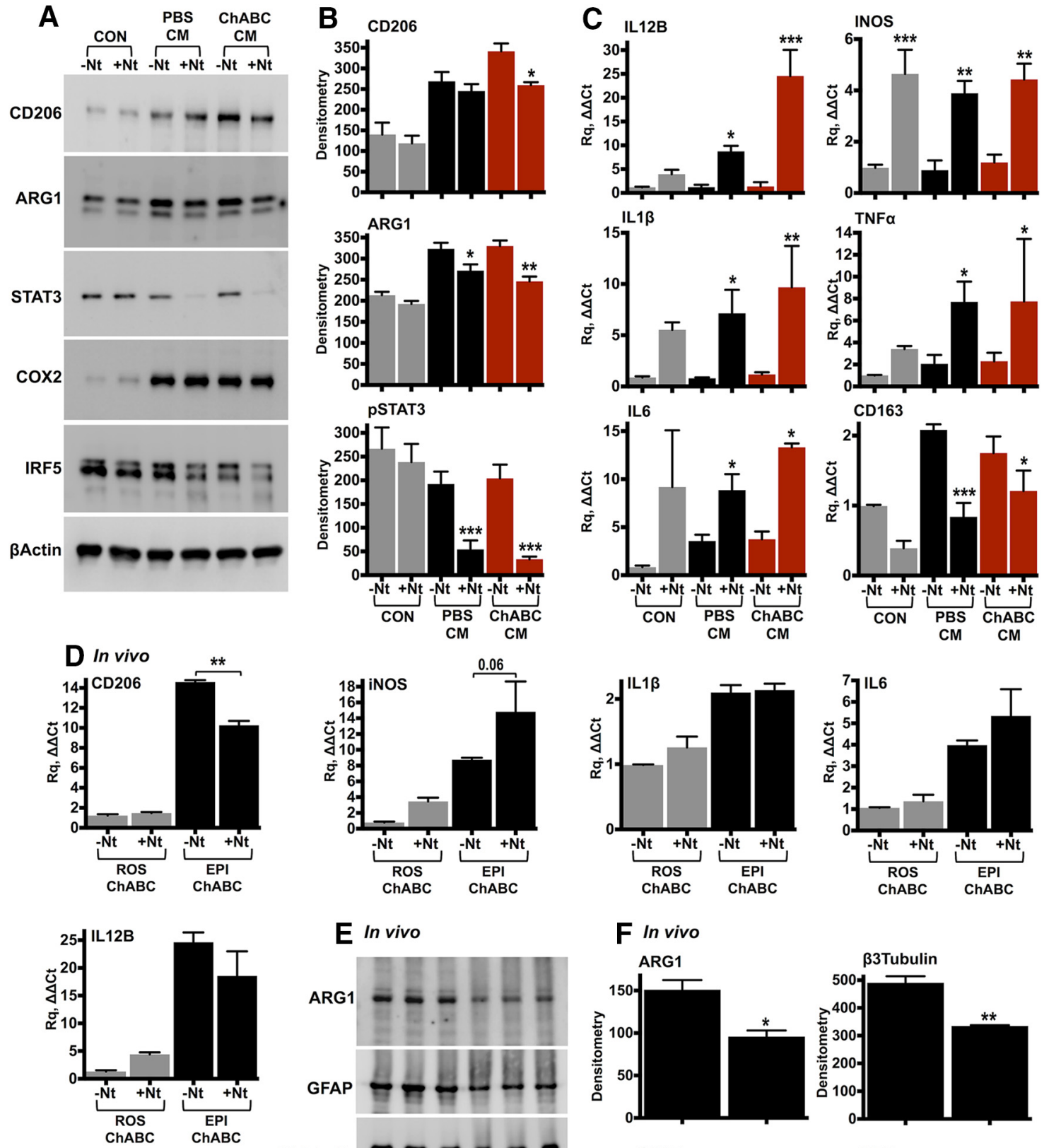

E In vivo

F In vivo
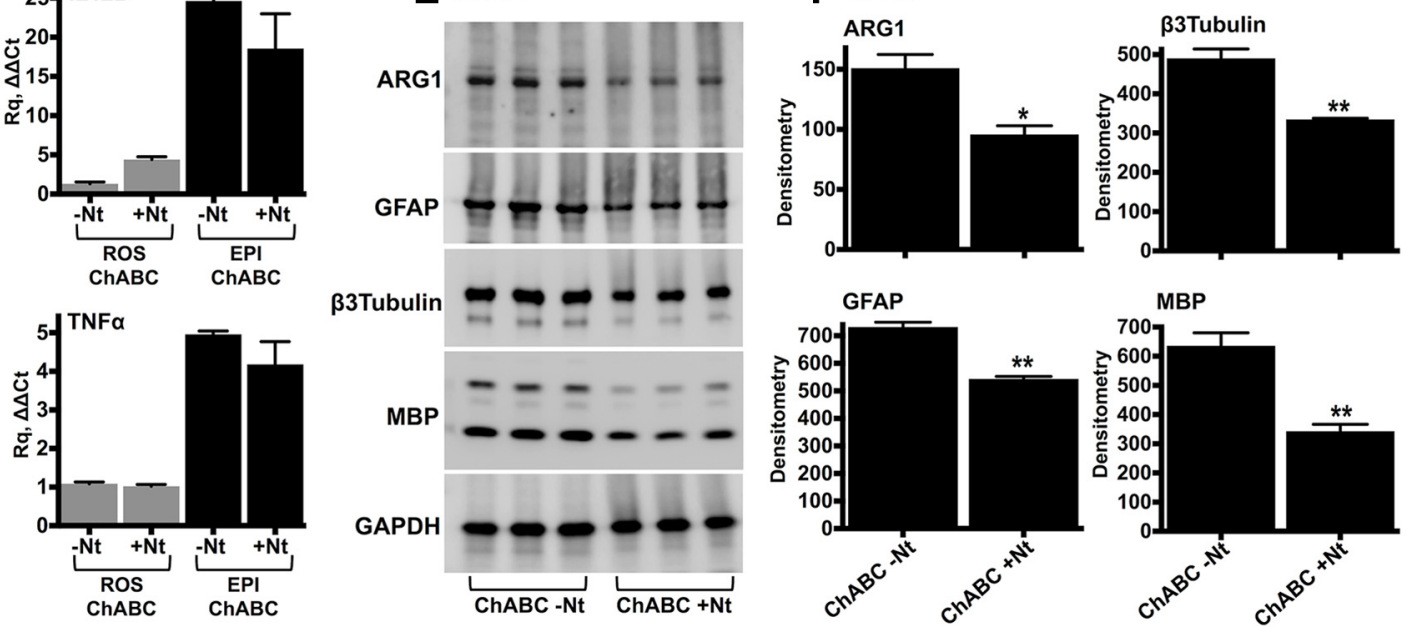

Figure 2. IL-10 neutralization in vitro and in vivo. $A, B$, Rat peritoneal macrophages were stimulated for $24 \mathrm{~h}$ with CM derived from the injury epicenter (T10) of either PBS- or ChABC-injected spinal cords. IL-10 was neutralized in the conditioned medium using anti-IL-10 antibodies ( $+\mathrm{Nt}, 250 \mathrm{ng}$ ). Control cells (CON) were cultured in serum-free DMEM, supplemented with either isotype rabbit $\lg \mathrm{G}(-\mathrm{Nt})$ or anti-IL-10 antibodies. Protein levels of inflammatory markers were assessed by immunoblotting $(\boldsymbol{A})$ and were quantified by densitometry $(\boldsymbol{B})$. Results are reported as the mean \pm SEM, $n=3-4$ experiments. $\beta$-Actin served as the loading control. C, The effect of IL-10 neutralization on the expression of inflammatory genes was assessed using TaqMan quantitative PCR in peritoneal macrophages stimulated with conditioned medium for $12 \mathrm{~h}$. The fold change in gene expression was calculated relative to unstimulated cells (CON, $-\mathrm{Nt}$ ). GAPDH served as the housekeeping gene. Results are the mean $\pm \mathrm{SEM}, n=3-4$ independent experiments. For $\boldsymbol{B}$ and $\boldsymbol{C}$, ANOVA and pairwise comparisons with Fisher's least significant difference test ( $-\mathrm{Nt}$ vs $+\mathrm{Nt}$ ). ${ }^{*} p<0.05,{ }^{* *} p<0.01,{ }^{* * *} p<0.001$. D-F, For in vivo neutralization of IL-10, T10 contused rats received intrathecal injections of ChABC ( $0.5 \mathrm{U} /$ injection) supplemented with either $5 \mu \mathrm{g}$ of isotype rabbit lgG (ChABC, $-\mathrm{Nt}$ ) or $5 \mu \mathrm{g}$ of anti-IL-10 neutralizing antibodies (ChABC, $+\mathrm{Nt}$ ). CD206, iNOS, IL-1 $\beta$, IL-6, IL-12B, and TNF- $\alpha$ expression was measured by quantitative PCR in the injury epicenter (EPI) and compared with intact rostral tissue (ROS) $7 \mathrm{~d}$ after $\mathrm{SCI}(\boldsymbol{D}) . n=3$ animals per group, ANOVA and Fisher's least significant difference test $(-\mathrm{Nt}$ vs $+\mathrm{Nt})$. ${ }^{* *} p<0.01 . \boldsymbol{E}, \boldsymbol{F}, \mathrm{ARG1}$, GFAP, $\beta 3$-tubulin, and MBP were examined in injured spinal cord protein extracts with immunoblotting $(\boldsymbol{E})$ and were quantified by densitometry $(\boldsymbol{F})$. Unpaired $t$ test, ${ }^{*} p<0.05$, ${ }^{* *} p<0.01$. GAPDH served as the loading control. 


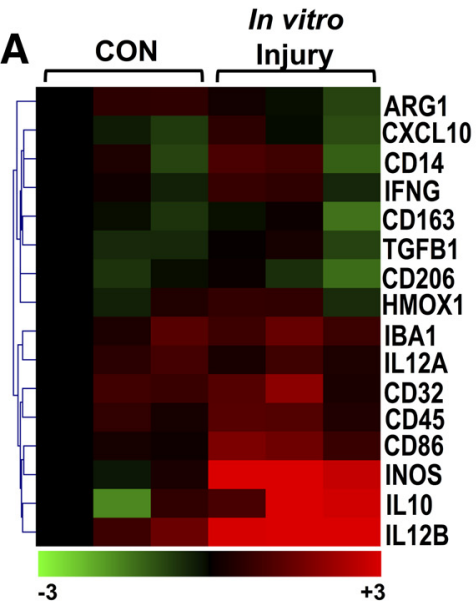

C

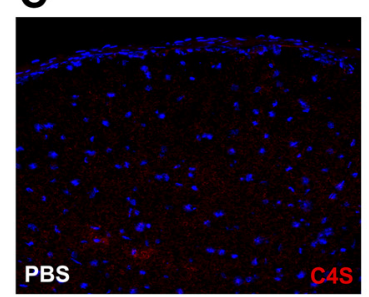

D
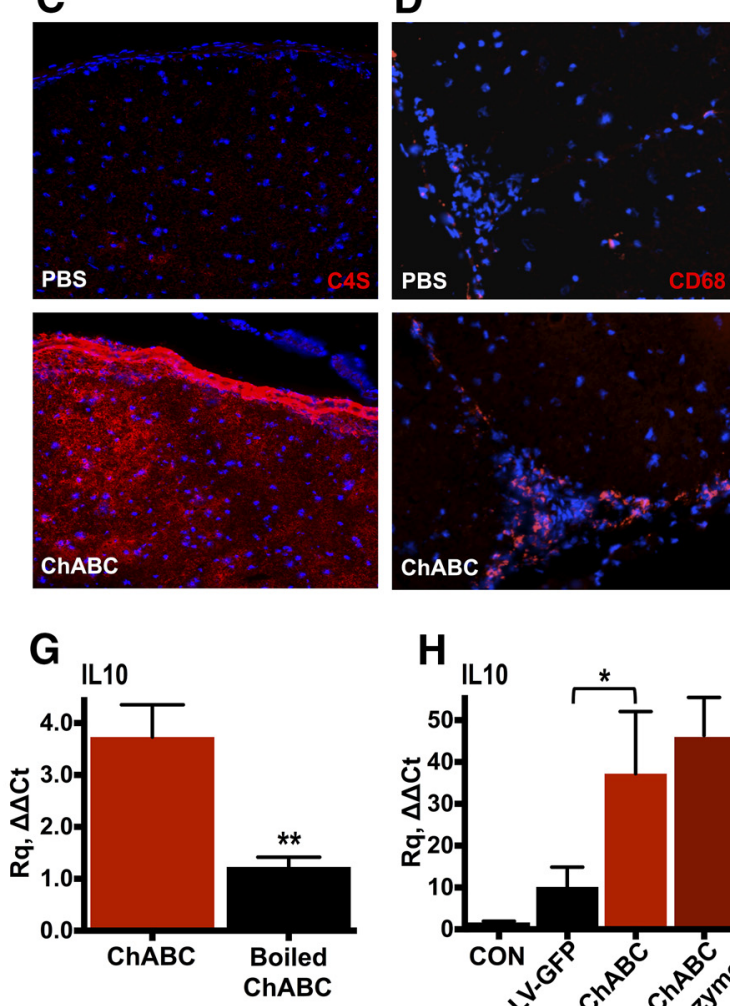
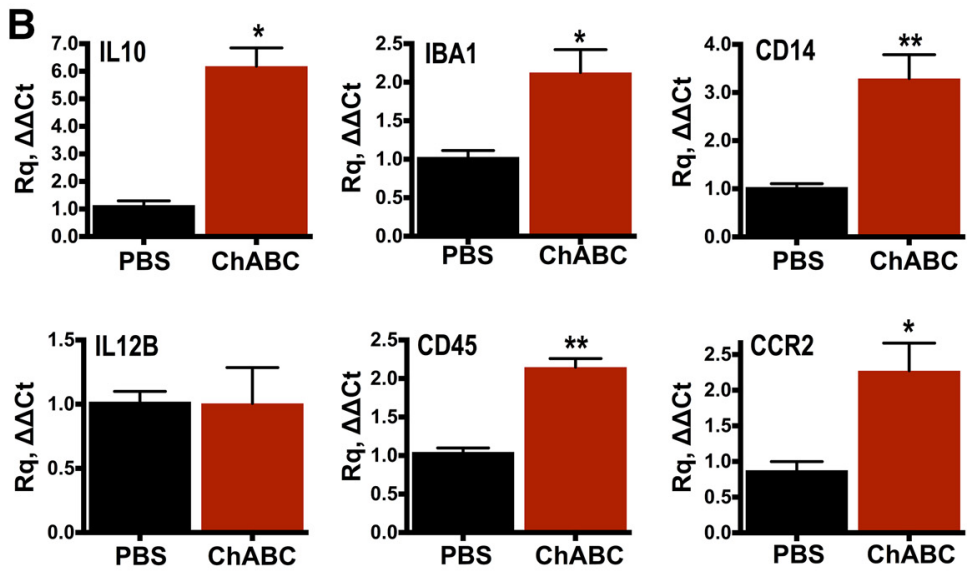

E
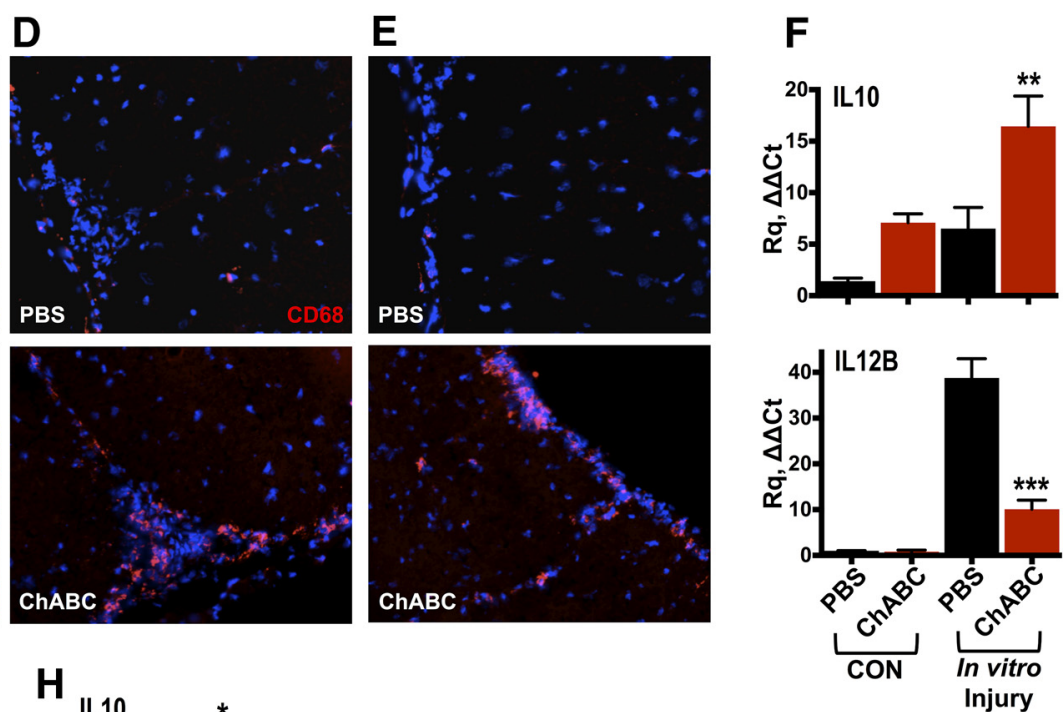

Figure 3. IL-10 expression is increased by in vitro injury and ChABC injections. $\boldsymbol{A}$, The heat map displays inflammatory gene expression measured by TaqMan quantitative PCR in T10 spinal cord explants dissected, diced, and cultured in vitro for $3 \mathrm{~h}$ (In vitro Injury). Baseline gene expression was measured in adjacent (T9) control explants that were snap frozen immediately after dissection (CON). $\boldsymbol{B}$, mRNA expression of inflammatory genes was measured in intact spinal cords after two intrathecal injections of either PBS or ChABC. Data are reported as the mean fold change \pm SEM, $n=$ 5 animals per group. Unpaired $t$ test, ${ }^{*} p<0.05,{ }^{* *} p<0.01$. C $-\boldsymbol{E}$, Spinal cords received intrathecal injections of either PBS or ChABC. Subsequently, T8 -10 cross-sections were immunostained with antibodies against C4S GAG stubs (C, red; scale bar, $100 \mu \mathrm{m})$ or CD68 (D, E, red; scale bar, $50 \mu \mathrm{m})$. Nuclei were counterstained using DAPI (blue). Note the presence of CD68-positive cells in the anterior spinal and sulcal vessels $(\boldsymbol{D})$ and the vasocorona $(\boldsymbol{E})$ of ChABC-injected cords. $\boldsymbol{F}$, Relative expression of IL-10 and IL-12B in T10 spinal cord explants dissected from uninjured animals that were preinjected with either PBS or ChABC. As in $A$, explants were cultured for $3 \mathrm{~h}$ (In vitro Injury). Baseline mRNA expression was determined in intact tissue (CON) collected from adjacent spinal cord segments, snap frozen immediately upon dissection. $n=4-5$ animals per group. ANOVA and Fisher's least significant difference test (PBS vs (hABC). ${ }^{* *} p<0.01,{ }^{* * *} p<0.001$. $\mathbf{G}, \mathrm{mRNA}^{*}$ expression of IL-10 in intact spinal cords after two intrathecal injections of either native ChABC or ChABC boiled for 15 min. $n=3$ animals per group. Unpaired $t$ test, ${ }^{* *} p<0.01$. $\boldsymbol{H}$, mRNA expression of IL-10 in the injury epicenter of contused spinal cords $7 \mathrm{~d}$ postinjury. Rats were treated with intraspinal injections of LVs expressing either GFP (LV-GFP) or ChABC (LV-ChABC). For comparison, other animals received intrathecal injections of bacterial ChABC. $n=3$ animals per group. ANOVA and Fisher's least significant difference test. * $p<0.05$.

While the upregulation of arginase 1 and CD163 and the downregulation of IL-12B could be attributed to increased IL-10 (Gordon and Martinez, 2010), the upregulation of iNOS was unexpected, indicating complex mechanisms of inflammatory balance. The specific regulation of IL-10 (high), IL-12B (low), iNOS (high), and arginase 1 (high) in contused ChABC-injected cords resembles the transcriptional profile of myeloid-derived suppressor cell, a population activated after trauma, suppressing $\mathrm{T}$-cell responses and inflammation in general (Gabrilovich and Nagaraj, 2009).

Consistent with our previous findings (Bartus et al., 2014), the M2 marker CD206 was upregulated by ChABC treatment. IL-10 


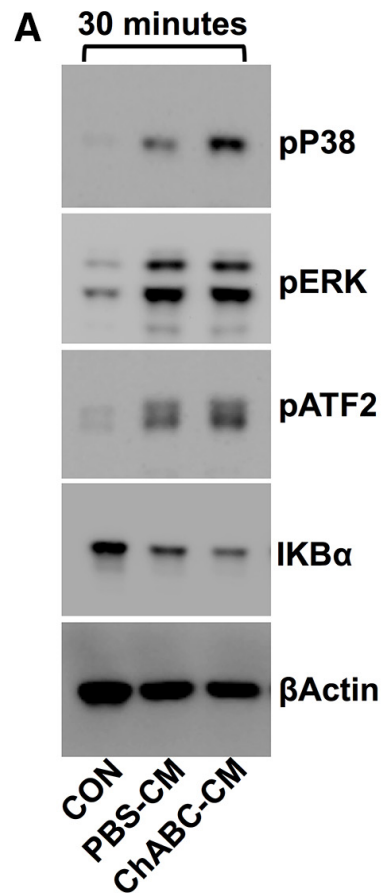

\section{Local inhibition of p38 (Intrathecal)}
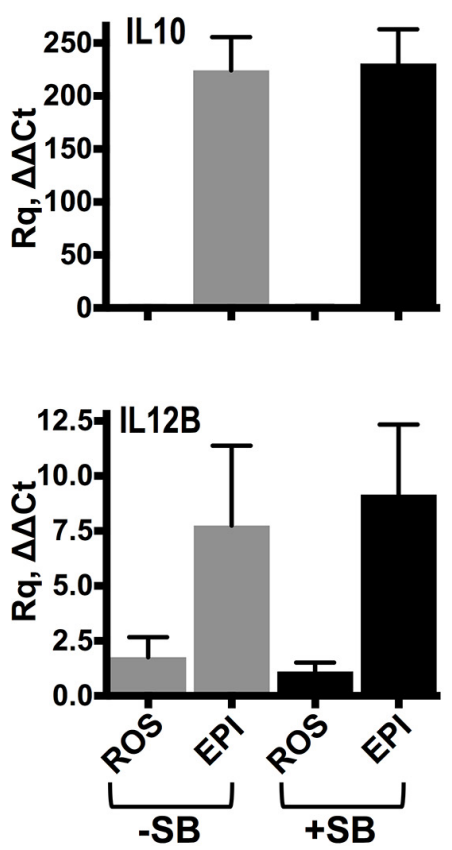

B
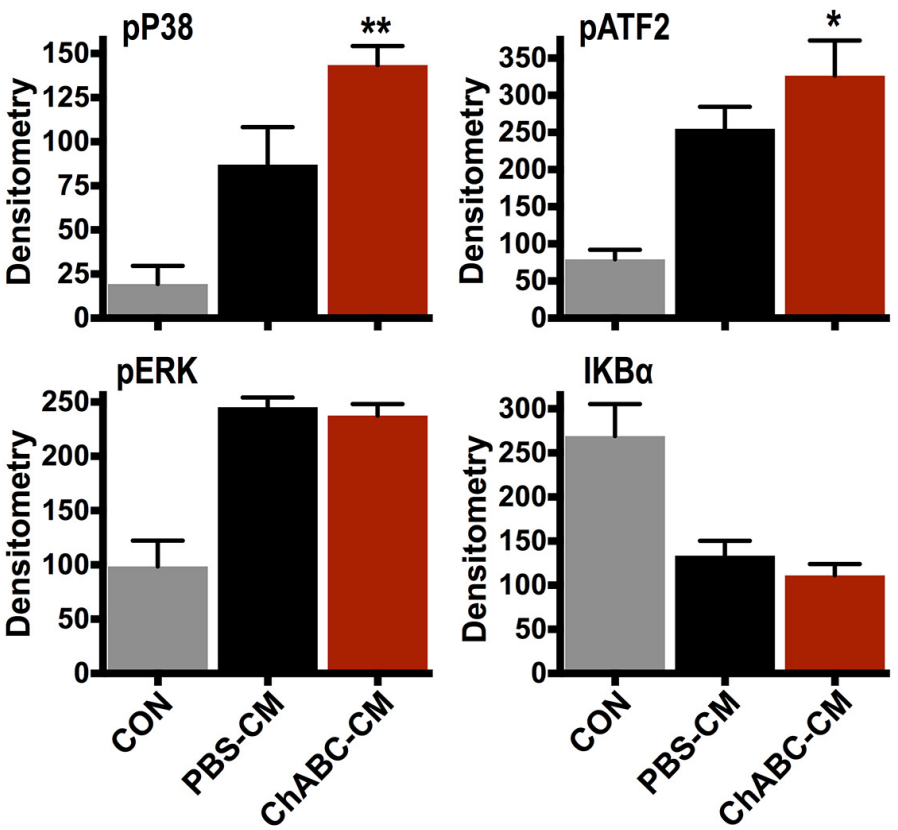

D Systemic inhibition of p38 (Intraperitoneal)
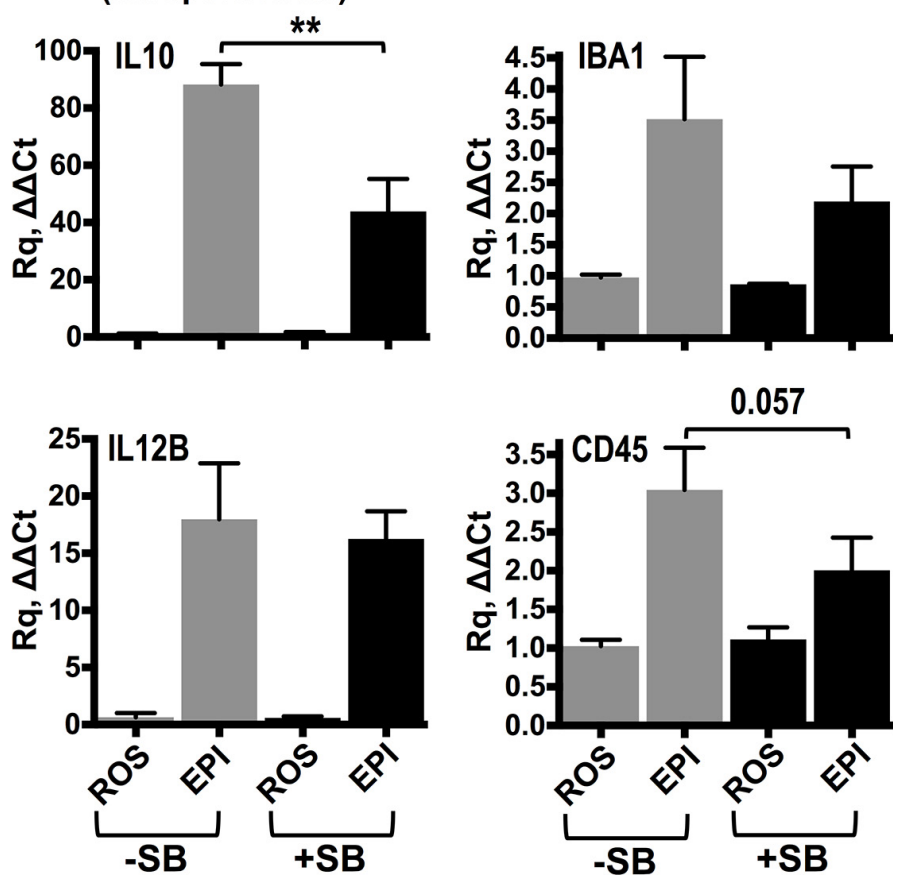

Figure 4. Molecular regulation of IL-10 by p38. $A, B$, Acute signaling activation was examined in lysates of peritoneal macrophages, stimulated for 30 min with CM derived from the injury epicenter of either PBS- or ChABC-injected spinal cords. Control cells (CON) were incubated with serum-free medium. Representative immunoblots from three independent experiments are shown $(\boldsymbol{A})$. Phosphoprotein and $I_{\kappa} \mathrm{B} \alpha$ levels were quantified by densitometry $(\boldsymbol{B})$. Values are reported as the mean density \pm SEM. ANOVA and Fisher's least significant difference test (PBS-CM vs ChABC-CM). ${ }^{*} p<0.05,{ }^{* *} p<0.01$. C, For spinal inhibition of p38, ChABC-injected rats received two intrathecal injections of either SB203580 (+ SB; $10 \mu m$ per injection) or vehicle (DMS0; - SB), 60 and 30 min before T10 contusion. IL-10 and IL-12B mRNA levels were measured by quantitative PCR in the injury epicenter (EPI, T10) and compared with intact rostral cord (R0S, T1). D, For systemic inhibition of $\mathrm{p} 38$, ChABC-injected rats received intraperitoneal injections of either SB203580 or vehicle (DMSO) before and immediately after T10 contusion. IL-10, IL-12B, IBA1, and CD45 mRNA levels were measured by qPCR in the injury EPI, $3 \mathrm{~h}$ after SCI. For $\boldsymbol{C}$ and $\mathbf{D}: n=3$ animals per group. ANOVA and Fisher's least significant difference test $(-$ SB vs $+\mathrm{SB}) .{ }^{* *} p<0.01$.

neutralization decreased CD206 in macrophages in vitro and in vivo after SCI, suggesting that IL-10 regulates CD206 expression in the injured spinal cord. Although the mannose receptor is classically controlled by IL-4, IL-10 can also induce it in macro- phages (Martinez-Pomares et al., 2003). In addition, IL-10 neutralization in vivo resulted in the reduction of GFAP, $\beta 3$-tubulin, and MBP, indicating a cytoprotective role of IL-10 in the injured spinal cord. Accordingly, Zhou et al. (2009) showed that viral 
overexpression of IL-10 is modestly neuroprotective after spinal hemisection, while Joniec-Maciejak et al. (2014) reported that IL-10 delivery increased GFAP expression in murine brains. Although here we focused on early inflammatory events after contusion injury, our biochemical findings on the immunomodulatory effects of high-concentration intrathecal ChABC and the increase in IL-10 raise important questions. Since we previously found that long-lasting and extensive deglycosylation of CSPGs achieved with lentiviral delivery of ChABC was associated with tissue repair and improved limb function after SCI (Bartus et al., 2014), it would be interesting to examine whether upregulation of IL-10 is maintained with long-term ChABC treatment and whether neutralizing IL-10 would prevent ChABC-dependent functional recovery.

Injection of high-dose ChABC also increased the expression of IL-10 in intact cords. The increase in IL-10 was associated with the upregulation of monocyte genes (CD45, CD14, and CCR2), while CD68-positive cells were found in the vasocorona of ChABC-injected spinal cords, suggesting monocyte infiltration. Interestingly, Shechter et al. (2009) showed that, after SCI in mice, infiltrating monocytes expressed IL-10, and that this was essential for immunoregulation and wound healing. Likewise, we showed that ChABC overexpression by lentiviral vectors is associated with influx of CD206-positive macrophages after SCI (Bartus et al., 2014). At present, the mechanism that governs the infiltration of macrophages in the cord after ChABC delivery is not known, but it is likely that enzymatic modification of the extracellular matrix drives this phenomenon. To this end, a study of nucleus pulposus resolution reported (Ishikawa et al., 1999) inflammatory infiltration in the epidural space of ChABCinjected animals. Importantly, we showed that the key immunomodulatory effects of ChABC (i.e., upregulation of CD206 and IL-10) are consistent between viral or intrathecal delivery of ChABC enzyme. However, not all of the observed biochemical changes are consistent; for example, here we show that $7 \mathrm{~d}$ postinjury IBA1 protein levels are slightly enhanced after high-dose intrathecal $\mathrm{ChABC}$ administration, in contrast to our previous observation that IBA1 was unchanged after intraspinal injection of ChABC-expressing virus (Bartus et al., 2014). This is perhaps due to differences in ChABC administration and/or enzymatic activity in the spinal cord. Another possibility that we cannot categorically exclude is that macrophages respond to the injection of the bacterial enzyme. Yet, the loss of IL-10 induction after boiling bacterial ChABC together with the increase in IL-10 after intraspinal injections of ChABC-expressing virus point toward a specific effect of ChABC activity on immunomodulation. We also found that IL-10 can be induced by simple in vitro mechanical injury of spinal cord explants, indicating that resident cells, most likely microglia and astrocytes (Ledeboer et al., 2002), can contribute to IL-10 expression in the cord and that IL-10 is an injury response gene, alongside IL-12B and iNOS.

Our data further revealed that p38 was the critical regulator of IL-10 expression. Systemic injection of the selective inhibitor SB203580 validated the role of p38 in postinjury induction of IL-10 in vivo and confirmed the involvement of infiltrating cells, since local administration of SB203580 via intrathecal injections before injury failed to reduce IL-10 mRNA. Although the regulation of IL-10 expression is complex and involves various pathways and transcription factors, p38 is a well established regulator of IL-10 in human monocytes and other cell types (Foey et al., 1998). Perhaps it is not surprising that p38 inhibition previously failed to alter pathological outcomes after SCI (Stirling et al.,
2008), reflecting the complexity of inflammatory regulation in vivo and the dominant involvement of p38 in both proinflammatory (IL-1, TNF) and anti-inflammatory (IL-10, tristetraprolin) mechanisms (Clark et al., 2009).

In summary, ChABC delivery alters inflammatory gene expression in the injured spinal cord. We report for the first time that the anti-inflammatory cytokine IL-10 is upregulated after ChABC treatment via a p38-dependent mechanism. The increased expression of IL-10 is linked to inflammatory regulation in the spinal cord and offers new insight into the beneficial effects of ChABC treatment after spinal cord injury.

\section{References}

Alilain WJ, Horn KP, Hu H, Dick TE, Silver J (2011) Functional regeneration of respiratory pathways after spinal cord injury. Nature 475:196-200. CrossRef Medline

Barritt AW, Davies M, Marchand F, Hartley R, Grist J, Yip P, McMahon SB, Bradbury EJ (2006) Chondroitinase ABC promotes sprouting of intact and injured spinal systems after spinal cord injury. J Neurosci 26:1085610867. CrossRef Medline

Bartus K, James ND, Didangelos A, Bosch KD, Verhaagen J, Yáñez-Muñoz RJ, Rogers JH, Schneider BL, Muir EM, Bradbury EJ (2014) Large-scale chondroitin sulfate proteoglycan digestion with chondroitinase gene therapy leads to reduced pathology and modulates macrophage phenotype following spinal cord contusion injury. J Neurosci 34:4822-4836. CrossRef Medline

Bradbury EJ, Moon LD, Popat RJ, King VR, Bennett GS, Patel PN, Fawcett JW, McMahon SB (2002) Chondroitinase ABC promotes functional recovery after spinal cord injury. Nature 416:636-640. CrossRef Medline

Burnside ER, Bradbury EJ (2014) Manipulating the extracellular matrix and its role in brain and spinal cord plasticity and repair. Neuropathol Appl Neurobiol 40:26-59. CrossRef Medline

Clark AR, Dean JL, Saklatvala J (2009) The p38 MAPK pathway mediates both antiinflammatory and proinflammatory processes: comment on the article by Damjanov and the editorial by Genovese. Arthritis Rheum 60: 3513-3514. CrossRef Medline

David S, Kroner A (2011) Repertoire of microglial and macrophage responses after spinal cord injury. Nat Rev Neurosci 12:388-399. CrossRef Medline

Fawcett JW, Asher RA (1999) The glial scar and central nervous system repair. Brain Res Bull 49:377-391. CrossRef Medline

Foey AD, Parry SL, Williams LM, Feldmann M, Foxwell BM, Brennan FM (1998) Regulation of monocyte IL-10 synthesis by endogenous IL-1 and TNF-alpha: role of the $\mathrm{p} 38$ and p42/44 mitogen-activated protein kinases. J Immunol 160:920-928. Medline

Gabrilovich DI, Nagaraj S (2009) Myeloid-derived suppressor cells as regulators of the immune system. Nat Rev Immunol 9:162-174. CrossRef Medline

Gordon S, Martinez FO (2010) Alternative activation of macrophages: mechanism and functions. Immunity 32:593-604. CrossRef Medline

Ishikawa H, Nohara Y, Miyauti S (1999) Action of chondroitinase ABC on epidurally transplanted nucleus pulposus in the rabbit. Spine (Phila Pa 1976) 24:1071-1076. CrossRef Medline

James ND, Bartus K, Grist J, Bennett DL, McMahon SB, Bradbury EJ (2011) Conduction failure following spinal cord injury: functional and anatomical changes from acute to chronic stages. J Neurosci 31:18543-18555. CrossRef Medline

Joniec-Maciejak I, Ciesielska A, Wawer A, Sznejder-Pachołek A, Schwenkgrub J, Cudna A, Hadaczek P, Bankiewicz KS, Członkowska A, Czønkowski A (2014) The influence of AAV2-mediated gene transfer of human IL-10 on neurodegeneration and immune response in a murine model of Parkinson's disease. Pharmacol Rep 66:660-669. CrossRef Medline

Kigerl KA, Gensel JC, Ankeny DP, Alexander JK, Donnelly DJ, Popovich PG (2009) Identification of two distinct macrophage subsets with divergent effects causing either neurotoxicity or regeneration in the injured mouse spinal cord. J Neurosci 29:13435-13444. CrossRef Medline 
Krausgruber T, Blazek K, Smallie T, Alzabin S, Lockstone H, Sahgal N, Hussell T, Feldmann M, Udalova IA (2011) IRF5 promotes inflammatory macrophage polarization and TH1-TH17 responses. Nat Immunol 12:231238. CrossRef Medline

Ledeboer A, Brevé JJ, Wierinckx A, van der Jagt S, Bristow AF, Leysen JE, Tilders FJ, Van Dam AM (2002) Expression and regulation of interleukin-10 and interleukin- 10 receptor in rat astroglial and microglial cells. Eur J Neurosci 16:1175-1185. CrossRef Medline

Mantovani A, Biswas SK, Galdiero MR, Sica A, Locati M (2013) Macrophage plasticity and polarization in tissue repair and remodelling. J Pathol 229:176-185. CrossRef Medline

Martinez-Pomares L, Reid DM, Brown GD, Taylor PR, Stillion RJ, Linehan SA, Zamze S, Gordon S, Wong SY (2003) Analysis of mannose receptor regulation by IL-4, IL-10, and proteolytic processing using novel monoclonal antibodies. J Leukoc Biol 73:604-613. CrossRef Medline

Saklatvala J, Rawlinson L, Waller RJ, Sarsfield S, Lee JC, Morton LF, Barnes MJ, Farndale RW (1996) Role for p38 mitogen-activated protein kinase in platelet aggregation caused by collagen or a thromboxane analogue. J Biol Chem 271:6586-6589. CrossRef Medline
Saraiva M, O'Garra A (2010) The regulation of IL-10 production by immune cells. Nat Rev Immunol 10:170-181. CrossRef Medline

Shechter R, London A, Varol C, Raposo C, Cusimano M, Yovel G, Rolls A, Mack M, Pluchino S, Martino G, Jung S, Schwartz M (2009) Infiltrating blood-derived macrophages are vital cells playing an anti-inflammatory role in recovery from spinal cord injury in mice. PLoS Med 6:e1000113. CrossRef Medline

Silver J, Miller JH (2004) Regeneration beyond the glial scar. Nat Rev Neurosci 5:146-156. CrossRef Medline

Stirling DP, Liu J, Plunet W, Steeves JD, Tetzlaff W (2008) SB203580, a p38 mitogen-activated protein kinase inhibitor, fails to improve functional outcome following a moderate spinal cord injury in rat. Neuroscience 155:128-137. CrossRef Medline

Williams LM, Sarma U, Willets K, Smallie T, Brennan F, Foxwell BM (2007) Expression of constitutively active STAT3 can replicate the cytokinesuppressive activity of interleukin-10 in human primary macrophages. J Biol Chem 282:6965-6975. CrossRef Medline

Zhou Z, Peng X, Insolera R, Fink DJ, Mata M (2009) IL-10 promotes neuronal survival following spinal cord injury. Exp Neurol 220:183-190. CrossRef Medline 